\title{
The measurement of relative efficiency using data envelopment analysis with assurance regions that link inputs and outputs
}

\author{
M. Khalili ${ }^{\mathrm{a}, \mathrm{b}, *}$, A.S. Camanho ${ }^{\mathrm{b}}$, M.C.A.S. Portela ${ }^{\mathrm{c}}$, M.R. Alirezaee ${ }^{\mathrm{a}}$ \\ ${ }^{a}$ Iran University of Science and Technology, Tehran, Iran \\ ${ }^{\mathrm{b}}$ Faculdade de Engenharia da Universidade do Porto, Portugal \\ ${ }^{\mathrm{c}}$ Universidade Católica Portuguesa, Porto, Portugal
}

Keywords:

Data envelopment analysis

Weight restrictions

Assurance regions type II

\section{A B S T R A C T}

The most popular weight restrictions are assurance regions (ARs), which impose ratios between weights to be within certain ranges. ARs can be categorized into two types: ARs type I (ARI) and ARs type II (ARII). ARI specify bounds on ratios between input weights or between output weights, whilst ARII specify bounds on ratios that link input to output weights. DEA models with ARI successfully maximize relative efficiency, but in the presence of ARII the DEA models may under-estimate relative efficiency or may become infeasible. In this paper we discuss the problems that can occur in the presence of ARII and propose a new nonlinear model that overcomes the limitations discussed. Also, the dual model is described, which enables the assessment of relative efficiency when trade-offs between inputs and outputs are specified. The application of the model developed is illustrated in the efficiency assessment of Portuguese secondary schools.

\section{Introduction}

The efficiency of decision making units (DMUs) in data envelopment analysis (DEA) is defined as the ratio of the weighted sum of outputs to the weighted sum of inputs. The weights are the variables of the DEA model, and DMUs have complete freedom to choose the weights associated with each input and/or output that maximise their relative efficiency. This complete flexibility in the selection of weights is especially important for identifying inefficient DMUs, as when the unit under assessment does not score $100 \%$ efficiency, this tells us that its peers are more productive even when the weights of all units are set to maximise the score of the unit assessed. Therefore, no inefficient unit can complain that its score would have been better if a different set of weights was used.

However, this complete flexibility may result in some inputs and/or outputs being assigned a zero or negligible weight, meaning that these factors are in fact ignored in the efficiency assessment. One way to limit the range of values that the weights can take is to use weight restrictions. Literature reviews on the use of weight restrictions in DEA can be found in Allen et al. (1997) and Thanassoulis et al. (2004).

Several types of weight restrictions (WRs) have been proposed in the DEA literature. In Allen et al. (1997) the direct weight restrictions are categorized into three types: assurance regions type I (first proposed by Thompson et al., 1986), assurance regions type II (first proposed by Thompson et al., 1990 and often called linked cone assurance regions), and absolute weight restrictions (first proposed by Dyson and Thanassoulis, 1988). Assurance regions (AR) are distinct from absolute weight restrictions because instead of imposing the weights to be within a certain range of values, they impose ratios between weights to be within certain ranges. ARI specify these ratios either between input or output weights separately, and ARII specify ratios that link input to output weights.

When absolute weight restrictions are imposed directly on DEA models with constant returns to scale (CRS) technology, the models may be infeasible, or the efficiency scores may be under-estimated (see e.g. Allen et al., 1997; Podinovski and Athanassopoulos, 1998). In order to obtain a correct estimate of relative efficiency in the presence of absolute weight restrictions, Podinovski and Athanassopoulos (1998) proposed the use of a Maximin model, and developed an equivalent linear programming formulation to enable an easy computation of relative efficiency scores, whilst avoiding all the problems of absolute weight restrictions (see also Podinovski, 1999).

The problems mentioned above do not occur in DEA models that use ARI (see Charnes et al., 1990; Thompson et al., 1990; Podinovski, 2001). This may explain the widespread use of this approach in DEA assessments (see e.g. Thompson et al., 1992 for oil/gas producers,

\footnotetext{
* Corresponding author. Address: Iran University of Science and Technology, Tehran, Iran. Tel.: +98 (0) 2177454199.

E-mail addresses: khalili26@yahoo.com (M. Khalili), acamanho@fe.up.pt (A.S. Camanho), csilva@porto.ucp.pt (M.C.A.S. Portela), mralirez@yahoo.com (M.R. Alirezaee).
} 
Schaffnit et al., 1997 for bank branches and Olesen and Petersen, 2002 for hospitals). However, similar problems occur in the presence of ARII, which may justify the small number of empirical applications that used DEA models with ARII (see e.g. Thanassoulis et al., 1995, that applied ARII for English Perinatal Care units). The use of ARII has been more frequent in "profit ratio models", which differ from standard DEA because the constraints imposing the ratio of virtual outputs to virtual inputs to be lower or equal to one are removed for all DMUs (see e.g. Thompson et al., 1995, 1996, 1997).

Thompson et al. (1990) and Thompson and Thrall (1994) pointed a limitation associated with the use of ARII in DEA models, which is the possibility of the model being infeasible for some or all DMUs. To overcome this limitation, Thompson and Thrall (1994) introduced a nonlinear DEA model that can retrieve the correct relative efficiency scores in the presence of ARII. As mentioned by the authors, the model was only solved for the special case of a single output and two inputs. Tracy and Chen (2005) also addressed this issue for a generalised form of weight restrictions, which encompass all forms of weight restrictions described in the literature. In fact, as explained in the next sections, our approach is similar to that of Tracy and Chen (2005) and solves some of its problems for the special case of ARII.

In this paper we explore the use of DEA models with ARII. The main limitations of these models, in addition to the infeasibility problem mentioned above, include the under-estimation of relative efficiency and the definition of a frontier for the production possibility set that may not include any of the observed DMUs. To overcome these limitations, inspired by the ideas of Thompson and Thrall (1994) and Podinovski and Athanassopoulos (1998), we propose the use of a nonlinear model that is equivalent to a Maximin model.

This paper is organized as follows. Section 2 explores the problems of using ARII associated to a standard formulation of the DEA model. The limitations of this procedure are illustrated using an example. Section 3 develops a new formulation of the DEA model that overcomes the problems described, and shows how the correct relative efficiency estimates can be retrieved in the presence of ARII. Section 4 applies the new model to Portuguese secondary schools. Section 5 concludes the paper.

\section{The interpretation of DEA effciency assessments with ARII}

The original DEA model for the estimation of relative efficiency was proposed by Charnes et al. (1978). The rationale of a relative efficiency measure is to compare the ratio output/input of the DMU assessed with the best value of this ratio observed in other DMUs. In the case of multiple input and multiple output assessments, the relative efficiency notion can be generalised to a comparison of ratios of the weighted sum of outputs to the weighted sum of inputs. To illustrate this idea, consider the assessment of $n$ DMUs $(j=1, \ldots, n)$. Each DMU uses $m$ inputs $x_{i j}(i=1, \ldots, m)$, to produce $s$ outputs $y_{r j}(r=1, \ldots, s)$. The input and output weights used for the efficiency assessment of a DMU are $v_{i}$ and $u_{r}$, respectively. The concept of relative efficiency can be defined using mathematical programming. The use of a Maximin model for this purpose was proposed by Thompson and Thrall (1994), and we reproduce in (1) the formulation described in Cooper et al. (1996).

$$
\max _{u, v}\left\{\left(\frac{\sum_{r} u_{r} y_{r o}}{\sum_{i} v_{i} x_{i o}}\right) /\left(\max _{j} \frac{\sum_{r} u_{r} y_{r j}}{\sum_{i} v_{i} x_{i j}}\right)\right\} .
$$

An equivalent fractional model can be obtained by defining $c=\max _{j}\left(\sum_{r} u_{r} y_{r j} / \sum_{i} v_{i} x_{i j}\right)$, such that model (1) becomes:

$$
\max _{u, v, c}\left\{\frac{1}{c}\left(\frac{\sum_{r} u_{r} y_{r o}}{\sum_{i} v_{i} x_{i o}}\right) \mid \frac{\sum_{r} u_{r} y_{r j}}{\sum_{i} v_{i} x_{i j}} \leqslant c, j=1, \ldots, n, u_{r} \geqslant 0 \forall r, v_{i} \geqslant 0 \forall i\right\} .
$$

Normalising the maximum ratio of weighted outputs to weighted inputs for all DMUs to 1 (i.e., defining $c=1$ ), the DEA model shown in ( 3 ) is obtained.

$$
\max _{u, v}\left\{\frac{\sum_{r} u_{r} y_{r o}}{\sum_{i} v_{i} x_{i o}} \mid \frac{\sum_{r} u_{r} y_{r j}}{\sum_{i} v_{i} x_{i j}} \leqslant 1, j=1, \ldots, n, u_{r} \geqslant 0 \forall r, v_{i} \geqslant 0 \forall i\right\} .
$$

The models above are equivalent to the linear programming formulations of the DEA model proposed by Charnes et al. (1978). The linear DEA output oriented model is reproduced in (4).

$$
\min _{u, v}\left\{\begin{array}{l}
\sum_{r} v_{i} x_{i o} \mid \sum_{i} u_{r} y_{r o}=1, \sum_{r} u_{r} y_{r j}-\sum_{i} v_{i} x_{i j} \leqslant 0, j=1, \ldots, n, \\
u_{r} \geqslant 0 \forall r, v_{i} \geqslant 0 \forall i
\end{array}\right\} .
$$

In models (1)-(4), the DMU under assessment is free to choose the weights that show it in the best possible light. Models (1) and (2) have no restrictions concerning the range of values admissible for the weights, so they are equivalent. In the case of model (3), there is an additional restriction imposing that none of the DMUs obtains a ratio of weighted outputs to weighted inputs higher than 1 . And in model (4) there is an additional limitation that the sum of weighted outputs is normalised to 1 . Models (1)-(4) have the same objective function value at the optimal solution in the absence of weight restrictions.

It is possible to add restrictions to the values of the weights, which can be expressed in different forms. Restrictions (5) and (6) correspond to ARI. Expression (5) defines relationships between input weights, and (6) defines relationships between output weights. Since the input and output weight restrictions are not linked, they define separate cones in the domain of the linear programming (LP) model (4). Hence, ARI can also be expressed as cone-ratios (see Charnes et al., 1990).

$$
\begin{aligned}
& \sum_{i} b_{i} v_{i} \leqslant 0, \\
& \sum_{r} a_{r} u_{r} \leqslant 0 .
\end{aligned}
$$


ARII define relationships between input and output weights (Thompson et al., 1990). The general formulation of ARII consisting on homogeneous inequality constraints is shown in (7). Note that at least a parameter $a_{r}$ and a parameter $b_{i}$ in restriction (7) must be nonzero, as otherwise it would become an ARI constraint.

$$
\sum_{r} u_{r} a_{r}-\sum_{i} v_{i} b_{i} \leqslant 0
$$

In the presence of ARII, models (1) and (2) give different efficiency estimates from models (3) and (4). If formulations (3) and (4) are used, the model may be infeasible and it is possible that none of the DMUs in the set assessed is considered fully efficient (100\% score). In addition, the efficiency estimates of formulations (3) and (4) are less than or equal to the estimates of models (1) and (2), which indicate that they may under-estimate the true relative efficiency value.

The general multiplier formulation of a DEA output oriented model with ARII under CRS is shown in (8).

$$
\min _{u, v}\left\{\begin{array}{l}
z_{o}=\sum_{i} v_{i} x_{i o} \mid \sum_{r} u_{r} y_{r o}=1, \sum_{r} u_{r} y_{r j}-\sum_{i} v_{i} x_{i j} \leqslant 0, j=1, \ldots, n, \\
\sum_{r} u_{r} a_{r t}-\sum_{i} v_{i} b_{i t} \leqslant 0, t=1, \ldots, k, u_{r} \geqslant 0 \forall r, v_{i} \geqslant 0 \forall i
\end{array}\right\} .
$$

The dual of model (8) is identical to a DEA model with trade-offs, as proposed by Podinovski (2004, 2005), and is shown in (9). In this model the ARII weight restrictions appear as trade-offs $\left(a_{r t}, b_{i t}\right)$ between inputs and outputs, showing the changes in outputs that would be expected from changes in inputs.

$$
\max _{\lambda, \pi, \theta}\left\{\begin{array}{l}
\theta \mid \sum_{j} \lambda_{j} x_{i j}+\sum_{t} \pi_{t} b_{i t} \leqslant x_{i o}, i=1, \ldots, m, \\
\sum_{j} \lambda_{j} y_{r j}+\sum_{t} \pi_{t} a_{r t} \geqslant \theta y_{r o}, r=1, \ldots, s, \pi_{t} \geqslant 0 \forall t, \lambda_{j} \geqslant 0 \forall j
\end{array}\right\} .
$$

In what follows, we discuss the limitations associated with the use of ARII or trade-offs in DEA models, which motivated the development of enhanced models that can correctly estimate relative efficiency when input and output weights are linked, or in the dual view when there are trade-offs between inputs and outputs (i.e., linked trade-offs). For that purpose consider a small illustrative example, consisting of 3 DMUs using one input to produce two outputs, as shown in Table 1.

The ARII imposed in the assessment of these DMUs are defined in (10). The ARII can be represented as equivalent trade-offs in the form $\left(a_{1 t}, a_{2 t}, b_{t}\right)$ as follows:

$$
\begin{aligned}
& \text { ARII Trade-offs } \\
& u_{1}-0.2 v \leqslant 0 \quad T_{1}:(1,0,0.2) \\
& \begin{array}{l}
0.1 \leqslant \frac{u_{1}}{v} \leqslant 0.2 \\
0.3 \leqslant \frac{u_{2}}{v} \leqslant 0.5
\end{array} \Longleftrightarrow \begin{array}{l}
-u_{1}+0.1 v \leqslant 0 \\
u_{2}-0.5 v \leqslant 0
\end{array} \Longleftrightarrow \begin{array}{l}
T_{2}:(-1,0,-0.1,) \\
T_{3}:(0,1,0.5)
\end{array} \\
& -u_{2}+0.3 v \leqslant 0 \quad T_{4}:(0,-1,-0.3)
\end{aligned}
$$

The first two elements in the trade-offs $\left(T_{t}\right)$ represent the change in the outputs that would be expected from a change in the input level equal to the value of the third element of the trade-off vector. A negative sign represents a decrease, and a positive sign represents an increase. For example, the first trade-off states that increasing the input by 0.2 would generate an increase in output $y_{1}$ by 1 , without changing output $y_{2}$. Solving model (7) or the trade-off model (8) with the ARII specified in (10) for the 3 DMUs considered yields the results shown in Table 2.

Results in Table 2 shows that only DMU B is efficient. Fig. 1 provides a graphical illustration of our example in the weights space.

The rectangle FGHI in Fig. 1 is the space defined by the ARII in (10). The feasible region of the LP model (7) is the shaded area in Fig. 1, defined as the intersection between the rectangle defined by the ARII and the space defined by the constraints of the form $\sum_{r} u_{r} y_{r j}-\sum_{i} v_{i} x_{i j} \leqslant 0$ specified for each DMU. The optimal solution of model (7) for DMUs A and B corresponds to point J, where

\begin{tabular}{|c|c|c|c|}
\hline DMU & $X$ & $Y 1$ & $Y 2$ \\
\hline A & 1 & 2 & 1 \\
\hline B & 1 & 1 & 2 \\
\hline $\mathrm{C}$ & 1 & 0.5 & 2 \\
\hline
\end{tabular}
$u_{1}=0.2$ and $u_{2}=0.4$. For DMU $C$ the optimal solution corresponds to point $K$, where $u_{1}=0.1$ and $u_{2}=0.45$ (see Table 2 ). Note that DMU B has alternative optimal solutions, corresponding to the points along the segment $\mathrm{KJ}$.

The efficiency estimates reported in Table 2 are not appropriate measures of relative efficiency, since the optimal weights in Table 2 do

\begin{tabular}{|c|c|c|c|c|}
\hline DMU & Efficiency $\left(1 / z_{o}^{*}\right)$ & $v^{*} / v^{*}$ & $u_{1}^{*} / v^{*}$ & $u_{2}^{*} / v^{*}$ \\
\hline A & 0.8 & 1 & 0.2 & 0.4 \\
\hline B & 1 & 1 & 0.2 & 0.4 \\
\hline C & 0.95 & 1 & 0.1 & 0.45 \\
\hline
\end{tabular}
not necessarily show the unit in the best possible light. To illustrate this, consider the assessment of DMU A. If we choose another com-

Table 1

Input and output data for the illustrative example.

Table 2

Optimal solution of model (7) with ARII (9) for all DMUs. 


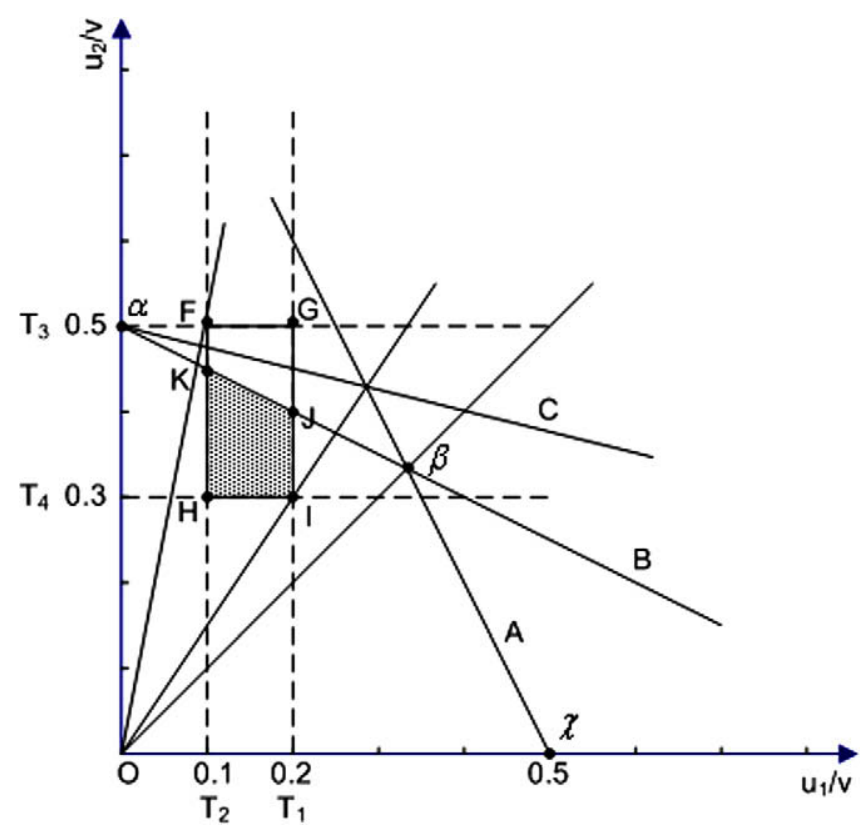

Fig. 1. Feasible region of model (7) with ARII (9) in the weights space.

bination of weights, with $v=1, u_{1}=0.2, u_{2}=0.3$, corresponding to point I in Fig. 1, the objective function of model (7) with restrictions (10) would be equal to 0.7 . This is clearly not the optimal solution to the model, as 0.7 is lower than 0.8 . However, with these weights, the highest efficiency value for all DMUs in the sample evaluated with these weights would be 0.8 related to DMU B. Therefore, the relative efficiency of DMU A would be equal to 0.875 as $0.7 / 0.8=0.875$. This example shows that a DEA model with ARII may overlook the set of weights most favourable for the assessment of relative efficiency.

Note that for an efficiency assessment using a DEA model without weight restrictions, the optimal weights for DMU C would be located at point $\alpha$, the optimal weights for assessing DMU B would be located in the segment between $\alpha$ and $\beta$, and the optimal weights for assessing DMU A would be located in the segment between $\beta$ and $\chi$. A similar relative efficiency score could be obtained using weights whose values were within the cone spanned by these segments and the origin. For example, any weight combination in the cone $\beta o \chi$ would render DMU A efficient. With this perspective, it becomes clear that for assessing the relative efficiency of DMU A, considering the weight constrains defined by the ARII represented in Fig. 1, the weight combination that shows this DMU in the best possible light corresponds to the vertex I of the feasible region. This example shows that a DEA model with ARII may overlook the set of weights most favourable for the assessment of relative efficiency, as the constraints of the DEA model (8) imply that the optimal solution of the LP must be located on the segment $\mathrm{KJ}$.

In addition, depending on the ARII defined it may also happen that no DMU reaches an efficiency score of $100 \%$. This would be the case if the second restriction defined in (10) was changed to $0.2 \leqslant \frac{u_{2}}{v} \leqslant 0.3$. In this case, a similar underestimation of relative efficiency would occur.

Fig. 2 is an alternative representation of the production possibility set, in the output space (where outputs have been normalised by the input), for the example in Fig. 1. In this figure the trade-offs (10) can be interpreted as new (unobserved) DMUs that were added to the production possibility set. The representation of these new DMUs in Fig. 2 implies a normalisation of the trade-offs to a unitary input, i.e., $T_{1}=(5,0,1), T_{2}=(-10,0,-1), T_{3}=(0,2,1)$ and $T_{4}=(0,-3.333,-1)$. Note that $T_{2}$ and $T_{4}$ can be equivalently represented as $T_{2}=(10,0,1)$ and $T_{4}=(0,3.333,1)$ provided that we divided all terms by -1 .

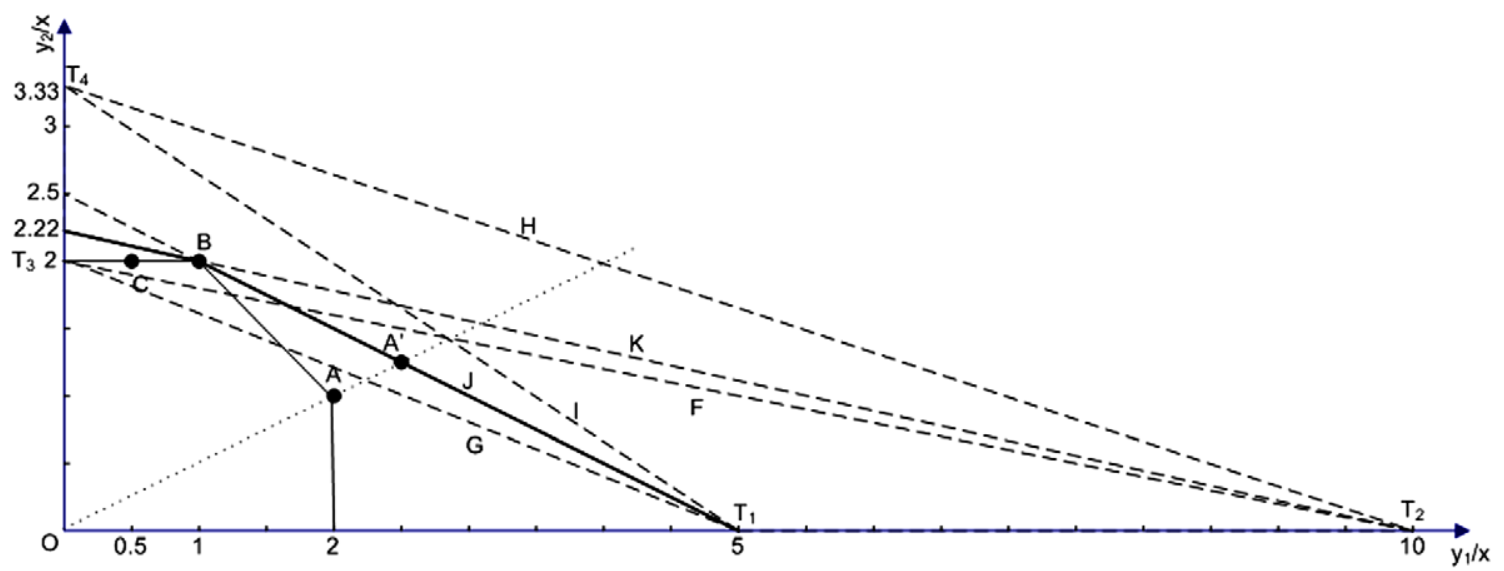

Fig. 2. The efficient frontier with and without ARII (9) in the input and output space. 


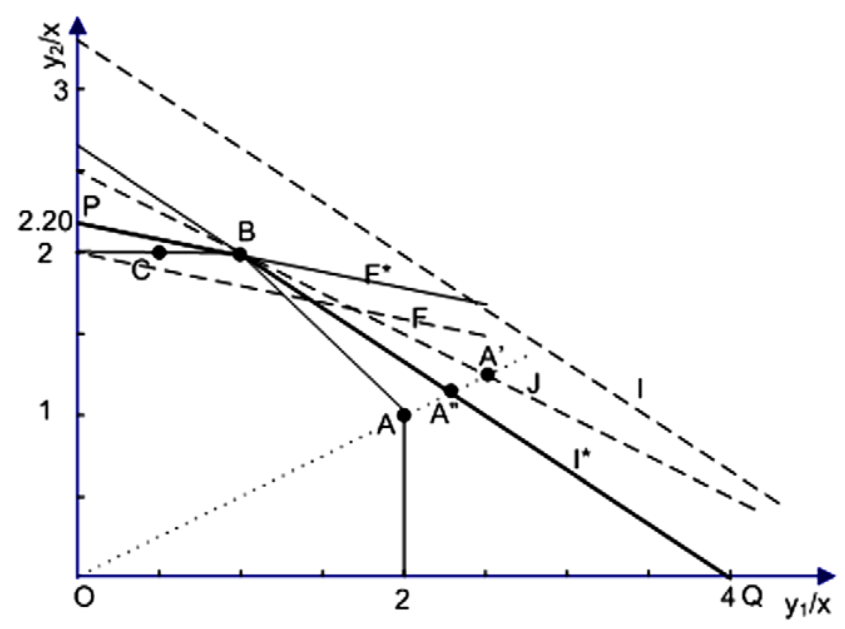

Fig. 3. Frontier for relative efficiency measurement.

The frontier of the production possibility set, without any WRs, is bounded by the lines connecting DMUs A, B and C, and its extensions to the axes. However, with the addition of the ARII defined in (10), the efficient frontier becomes the bold lines spanning from point B. This frontier is constituted by segment $\mathrm{K}$ to the left of DMU B, and by segment J to the right of DMU B. Note that line segments in Fig. 2 correspond to vertices in Fig. 1, obtained from the expression $\sum_{r} u_{r}^{*} y_{r}-\sum_{i} v_{i}^{*} x_{i}=0$ in each point $\left(u_{r}^{*}, v_{i}^{*}\right)$ in Fig. 1 . Segment K is the line connecting the unobserved DMU generated from trade-off $T_{2}$ and the observed DMU B (corresponding to point K in Fig. 1 ). Segment J is the line connecting the unobserved DMU generated from trade-off $T_{1}$ and the observed DMU B (corresponding to point $\mathrm{J}$ in Fig. 1 ). All the remaining lines drawn correspond to the points identified in Fig. 1, and are either defined through the connection of unobserved DMUs only, (as it is the case of line $\mathrm{H}, \mathrm{I}, \mathrm{F}, \mathrm{G}$ ) or through the connection of observed DMUs and unobserved DMUs (as it is the case of lines $\mathrm{K}$ and J). The assessment of DMU A in relation to this frontier using a standard DEA model with the ARII would imply its comparison with point $A^{\prime}$. The efficiency score of DMU A would be equal to $\mathrm{OA} / \mathrm{OA}^{\prime}=0.8$. Point $\mathrm{A}^{\prime}$ can be regarded as the target for DMU A if we consider that the trade-offs defined represent feasible modifications of the operating conditions observed at DMU $B$. In this circumstance we can regard the trade-offs as extending the original PPS (the area below CBA) to the area below the frontier represented by the bold line in Fig. 2 . Note however, that the trade-offs may in fact imply that none of the observed DMUs is considered $100 \%$ efficient, and under that circumstance the trade-offs would not extend the original PPS but would create a completely new one.

There are therefore some reasons for not accepting the above trade-offs as reasonable ones. The most important reason is the fact that the frontier defined by the bold lines in Fig. 2 under-estimates relative efficiency. This is because the efficient frontier constructed from observed and non-observed DMUS is defined as the closest to the original PPS. If we interpret the trade-offs as marginal rates of substitution between inputs and outputs, then all slopes such as those exemplified in Fig. 2 by the dotted lines (and in general all slopes that correspond to the cone IOF from Fig. 1) could be used in the relative efficiency assessment. Therefore, to assess the DMUs in the best possible light, instead of using lines $\mathrm{K}$ and $\mathrm{J}$ from Fig. 2, we could use the slopes of lines I and $\mathrm{F}$, and this could lead to the construction of the new frontier shown in Fig. 3. The frontier is obtained by moving lines I and F until they become tangent to the PPS. Unit A would, therefore, be radially projected to point $A^{\prime \prime}$ in Fig. 3 rather than to point $A^{\prime}$ in Fig. 2, and would be rated more efficient than before. The next section describes the modification required to the DEA model in order to evaluate relative efficiency in the presence of ARII, adopting the conceptual framework illustrated in Fig. 3.

\section{A new model for estimating relative effciency with ARII}

As mentioned by Thompson and Thrall (1994) and Tracy and Chen (2005) to retrieve the relative efficiency scores of a DEA model with ARII one needs to use a Maximin model. However, to date there is not a standard procedure on how to estimate relative efficiency scores in these circumstances because the Maximin model with ARII is nonlinear. Also Podinovski's (2004) trade-off model (8) cannot assess the maximum relative efficiency in the presence of linked trade-offs as shown in the previous section.

In order to put forward a new model for measuring relative efficiency in the presence of ARII, we first note that in traditional DEA models (like (3) or (4)) the imposition that the efficiency score cannot exceed the value of 1 (or $100 \%$ ) is just a convention since we could state that the efficiency should not exceed any arbitrary positive number $c$, as shown in model (2) (see also Tracy and Chen, 2005). The choice of the value of $c$ is irrelevant for assessments without weight restrictions, or with ARI. However, when ARII are added to the standard fractional model (2), the efficiency scores obtained for each DMU are dependent on the value of $c$. However, the value of $c$ that leads to maximum efficiency is not known a-priori. To be able to assess each DMU with the most favourable value of $c$, we propose the use of the nonlinear model (11), where $c$ is considered as a variable rather than a parameter. Model (11) is the appropriate formulation for estimating relative efficiency in the presence of ARII restrictions.

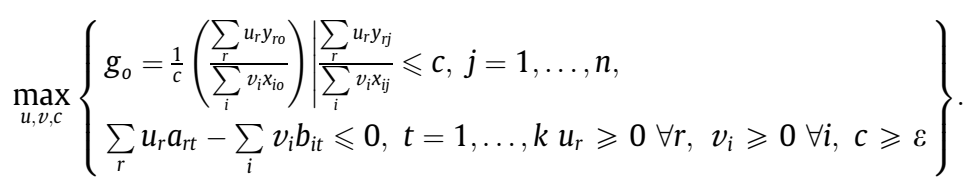

To remove the fractional terms, model (11) can be written as (12) for an input oriented assessment (see proof in the Appendix A). 


$$
\max _{u, v, c}\left\{\begin{array}{l}
g_{o}=\sum_{r} u_{r} y_{r o} \mid c \sum_{i} v_{i} x_{i o}=1, \sum_{r} u_{r} y_{r j}-c \sum_{i} v_{i} x_{i j} \leqslant 0, j=1, \ldots, n \\
\sum_{r} u_{r} a_{r t}-\sum_{i} v_{i} b_{i t} \leqslant 0, t=1, \ldots, k, u_{r} \geqslant 0, v_{i} \geqslant 0, c \geqslant \varepsilon
\end{array}\right\} .
$$

For an output oriented assessment model (13) should be used.

$$
\min _{u, v, c}\left\{\begin{array}{l}
h_{o}=\sum_{i} v_{i} x_{i o} \mid c \sum_{r} u_{r} y_{r o}=1, c \sum_{r} u_{r} y_{r j}-\sum_{i} v_{i} x_{i j} \leqslant 0, j=1, \ldots, n \\
\sum_{r} u_{r} a_{r t}-\sum_{i} v_{i} b_{i t} \leqslant 0, t=1, \ldots, k, u_{r} \geqslant 0, v_{i} \geqslant 0, c \geqslant \varepsilon
\end{array}\right\} .
$$

Models (12) and (13) are nonlinear (NL) models, but for the special cases of one single input and one single output, respectively, these models become linear.

Note that the efficiency estimates obtained from the input oriented model (12) or output oriented model (13) coincide for CRS, such that $g_{o}=1 / h_{0}$. The results of the output oriented model (13) for the DMUs of our illustrative example with the ARII (10) are shown in Table 3, where all input and output weights have been normalised by the input weight. As can be seen in Table 3, DMU B is efficient, and the relative efficiency of the remaining DMUs is higher than that reported in Table 2.

Assuming that the value of the variable $c$ at the optimal solution to (13) is known, it is possible to provide a dual formulation for model (13), treating $c$ as a parameter and using linear programming duality theory. Note that we describe the output oriented model in detail, but the construction of the input oriented version is straightforward. Fixing variable $c$ in model (13) with the corresponding optimal value, the dual model (14) is obtained using linear programming duality theory:

$$
\max _{\lambda, \pi, \beta_{o}}\left\{\begin{array}{l}
\beta_{o} \mid \sum_{j} \lambda_{j} x_{i j}+\sum_{t} \pi_{t} b_{i t} \leqslant x_{i o}, i=1, \ldots, m, \\
c^{*} \sum_{j} \lambda_{j} y_{r j}+\sum_{t} \pi_{t} a_{r t} \geqslant c^{*} \beta_{o} y_{r o}, r=1, \ldots, s, \pi_{t} \geqslant 0, \lambda_{j} \geqslant 0
\end{array}\right\} .
$$

Model (14) can be interpreted as a modified trade-offs model, which correctly estimates relative efficiency in the presence of linked tradeoffs. To construct model (14) $c$ was treated as a parameter obtained from the optimal solution of NL model (13). We can interpret the value of $c^{*}$ in model (14) as a scaling factor of the outputs. Fig. 4 shows the frontier underlying the assessment with model (14), in the output space, for DMU A.

The graphical representation in Fig. 4 scales outputs by $c$ as dictated by the constraints in model (14). The frontier in Fig. 4 (the darker line) is that used to evaluate DMU A, where the optimal $c$ is 1.25 . For example, the relative efficiency of DMU A will be assessed by the ratio of $\mathrm{OA}^{*} / \mathrm{OA}^{\prime \prime}$, being equal to 0.875 as reported in Table 3. This measure is identical to the ratio OA/OA" illustrated in Fig. 3 (but in this figure it was the trade-off that was scaled rather than the outputs - as will be clear afterwards these two approaches are equivalent).

Fig. 4 illustrates one of the possible interpretations of the relative efficiency estimation in the presence of ARII. The original PPS is scaled up or down such that it touches the line corresponding to the trade-off with the most favourable slope for the evaluation of the DMU.

It is possible to provide alternative interpretations for the relative efficiency measure by applying some variable substitutions to (14). Dividing the output restrictions of model (14) by $c^{*}$ and defining $\gamma^{*}=1 / c^{*}$, model (15) is obtained. Therefore, we can interpret the output

\begin{tabular}{|c|c|c|c|c|c|}
\hline DMU & Efficiency $\left(1 / h_{o}^{*}\right)$ & $c^{*}$ & $v^{*} / v^{*}$ & $u_{1}^{*} / v^{*}$ & $u_{2}^{*} / v^{*}$ \\
\hline A & 0.875 & 1.25 & 1 & 0.2 & 0.3 \\
\hline B & 1 & 1.25 & 1 & 0.2 & 0.3 \\
\hline $\mathrm{C}$ & 0.9545 & 0.909 & 1 & 0.1 & 0.5 \\
\hline
\end{tabular}

Table 3

Optimal solution of model (12) for the illustrative example.

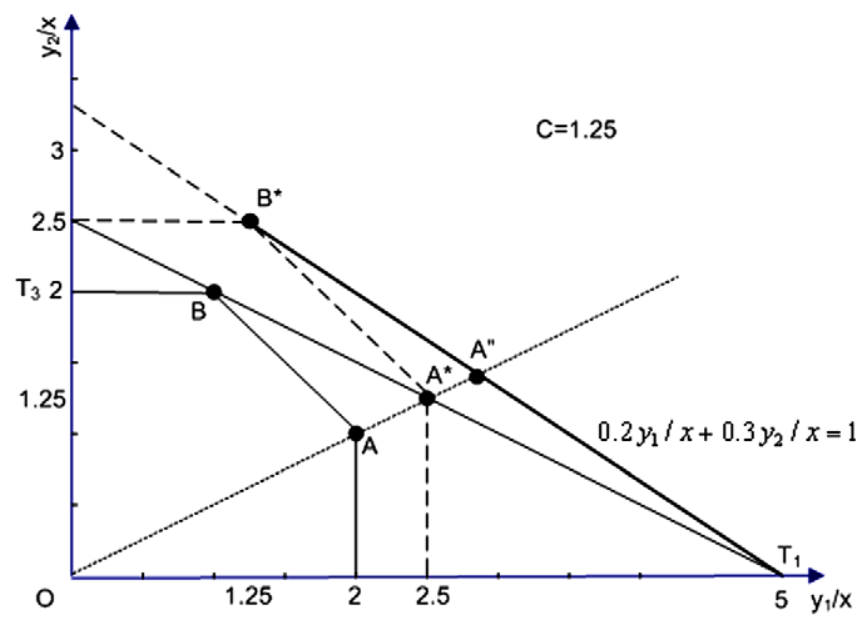

Fig. 4. The PPS and efficient frontier for assessing DMU A. 
constraints in (14) as having a scaling factor $c^{*}$ associated to observed output levels (which led to our Fig. 4), or equivalently, a scaling factor $\gamma^{*}=1 / c^{*}$ associated to the trade-offs, as illustrated before in Fig. 3 .

$$
\max _{\lambda, \pi, \beta_{0}, \gamma}\left\{\begin{array}{l}
\beta_{0} \mid \sum_{j} \lambda_{j} x_{i j}+\sum_{t} \pi_{t} b_{i t} \leqslant x_{i o}, i=1, \ldots, m, \\
\sum_{j} \lambda_{j} y_{r j}+\gamma^{*} \sum_{t} \pi_{t} a_{r t} \geqslant \beta_{0} y_{r o}, r=1, \ldots, s, \pi_{t} \geqslant 0, \lambda_{j} \geqslant 0, \gamma \geqslant 0
\end{array}\right\} .
$$

The frontier in Fig. 3 is represented by the bold line PBQ, where $Q$ is the trade-off $(5,0,1)$ scaled to $(4,0,1)$ and $P$ is the trade-off $(0,2,1)$ scaled to $(0,2.2,1)$. Fig. 3 shows that the frontier is arrived at by scaling the appropriate line $\mathrm{I}\left(0.2 y_{1} / x+0.3 y_{2} / x=1\right)$ down by 0.8 and scaling the line $\mathrm{F}\left(0.1 y_{1} / x+0.5 y_{2} / x=1\right)$ up by 1.1 . Fig. 3 allows one to show that, similarly to the Podinovski (2004) trade-off model, our nonlinear model provides a radial measure of efficiency, computed in relation to a modified frontier as dictated by the introduction of the ARII. This can be seen easily through model (15), where the radial output targets would correspond to the right hand side of the output constraint, which increments all the original output levels by the same factor $\beta_{0}$.

Targets that remove both radial and non-radial inefficiencies can be obtained using expressions (16), where $x_{i}^{T}$ represents the input targets and $y_{t}^{T}$ represents the output targets.

$$
\begin{aligned}
& x_{i}^{T}=\sum_{j} \lambda_{j}^{*} x_{i j}+\sum_{t} \pi_{t}^{*} b_{i t} \quad \forall i, \\
& y_{t}^{T}=\sum_{j} \lambda_{j}^{*} y_{r j}+\gamma^{*} \sum_{t} \pi_{t}^{*} a_{r t} \quad \forall r .
\end{aligned}
$$

Computing targets using (16) for unit $\mathrm{A}\left(y_{1}, y_{2}, x\right)=(2,1,1)$, for example, yields $\left(y_{1}^{T}, y_{2}^{T}, x^{T}\right)=(2 \times 1 / 0.875,1 / 0.875,1)=(2.286,1.143,1)$, corresponding to point $\mathrm{A}^{\prime \prime}$ in Fig. 3.

In terms of computational requirements, the nonlinear solvers available in GAMS could solve models (13), (14) or (15) and obtain the global optimal solution for our illustrative example and empirical application. The solvers MINOS, CONOPT, SNOPT and PATHNLP were tested, and all provided the correct optimal solution to the NL model. Since these models are non-convex, it cannot be guaranteed that a nonlinear solver will always be able to find the global optimal solution. It is therefore advisable to use nonlinear solvers that have procedures to find the global optimal solution for non-convex nonlinear models. For example, using the software GAMS, the solvers BARON, LGO and OQNLP would also be recommended. We compared the results of our nonlinear model (13) with the results of a neighbourhood search algorithm proposed by Tracy and Chen (2005). The higher relative efficiency estimates obtained solving our nonlinear model confirmed that the results of the nonlinear model are global optimum.

\section{Empirical application to a sample of secondary schools}

This section describes the efficiency assessment of 18 secondary schools in Portugal using the data set of Portela and Camanho (2007). Secondary education in Portugal happens for a period of 3 years, and the data analysed corresponds to the cohort of pupils that started secondary education in the academic year 2002/2003 and finished in 2004/2005. Table 4 summarizes the input and output variables used in the DEA model. The input variables characterize the student cohort on entry of secondary education, and the outputs reflect academic achievement on exit.

Table 5 shows the input and output data of the schools analysed, and the summary statistics for the sample.

In establishing the weight restrictions we used a method based on trade-off information. This is an indirect and interactive method used in Multiple-Criteria Decision Analysis to help a decision maker express preference judgments concerning the relative importance of the variables used in the model (Belton and Stewart, 2002). Interactive methods lead decision makers, through a sequence of simple choices, to reach a vector, corresponding to an unobserved DMU, which is considered equivalent to an original DMU. With the identification of indifferent schools in terms of performance, we are able to specify indirectly the value judgments of the decision maker concerning how the change in the input set should impact the change in the output set for an efficient school to remain at the frontier. This type of reasoning is the underlying idea for defining weight restrictions in DEA models based on trade-offs, as proposed by Podinovski (2004).

Therefore we analysed the average school and asked an expert to provide values for the variables that made the average school and the 'changed' school indifferent in terms of performance. In order to do this, we did not use the average values in volume but in ratio per student on entry (i.e., all variables were divided by the input number of students on entry). In doing so, the average school presents the values in Table 6, where we also show the perturbations in some variables that constitute indifferent schools.

The implicit trade-offs in these indifferent schools are as follows (see Table 7).

The trade-offs can be converted into equivalent ARII, which are shown in (17).

$$
\begin{aligned}
& u_{1}-v_{2} \leqslant 0 \\
& 0.02 u_{2}+0.27 u_{1}-v_{2} \leqslant 0 \\
& -0.57 u_{1}+v_{3} \leqslant 0 \\
& -0.05 u_{2}+u_{1} \leqslant 0
\end{aligned}
$$

\begin{tabular}{|c|c|}
\hline Inputs & Outputs \\
\hline $\begin{array}{l}\text { - Number of pupils on entry } \\
\text { - Scores on entry } \\
\text { - Number of years in school for the parents }\end{array}$ & $\begin{array}{l}\text { - Scores on exit on national exams } \\
\text { - Students that completed secondary } \\
\text { - Education in } 3 \text { years }\end{array}$ \\
\hline
\end{tabular}

Table 4

Inputs and outputs for the assessment of secondary schools. 
Table 5

Inputs and outputs data of secondary schools.

\begin{tabular}{|c|c|c|c|c|c|}
\hline DMU & No. of pupils on entry & Scores on entry & No. of years in school for the parents & Scores on exit on national exams & Students that completed SE in 3 years \\
\hline 1 & 240 & 2747.70 & 2785.20 & 2874.07 & 84 \\
\hline 2 & 410 & 4427.86 & 4179.95 & 4081.60 & 116 \\
\hline 3 & 192 & 1990.01 & 1926.72 & 2155.10 & 57 \\
\hline 4 & 149 & 1575.80 & 1554.07 & 1714.02 & 59 \\
\hline 5 & 41 & 468.08 & 575.44 & 432.48 & 20 \\
\hline 6 & 272 & 2716.76 & 2696.88 & 3017.56 & 93 \\
\hline 7 & 161 & 1646.48 & 1589.88 & 1526.03 & 42 \\
\hline 8 & 294 & 3216.01 & 3136.98 & 3504.91 & 135 \\
\hline 9 & 84 & 802.58 & 881.58 & 804.61 & 25 \\
\hline 10 & 143 & 1475.96 & 1568 & 1546.40 & 41 \\
\hline 11 & 482 & 5473.14 & 6037.05 & 6271.14 & 281 \\
\hline 12 & 234 & 2480.74 & 2283.84 & 2546.29 & 63 \\
\hline 13 & 101 & 1117.20 & 991.82 & 1023.38 & 35 \\
\hline 14 & 673 & 7092.79 & 6770.38 & 7418.01 & 155 \\
\hline 15 & 161 & 1661.75 & 1651.86 & 1532.46 & 49 \\
\hline 16 & 147 & 1575.09 & 1381.80 & 1701.08 & 50 \\
\hline 17 & 165 & 1807.22 & 1645.05 & 1855.84 & 75 \\
\hline 18 & 162 & 1738.35 & 1751.22 & 1823.20 & 58 \\
\hline average & 228.39 & 2445.20 & 2411.54 & 2546.01 & 79.89 \\
\hline $\max$ & 673 & 7092.80 & 6770.38 & 7418.01 & 281 \\
\hline $\min$ & 41 & 468.08 & 575.44 & 432.48 & 20 \\
\hline
\end{tabular}

Table 6

Average and indifferent schools.

\begin{tabular}{|c|c|c|c|c|}
\hline & $\begin{array}{l}\text { Average scores on } \\
\text { entry }\end{array}$ & $\begin{array}{l}\text { Average no. of years in school for the } \\
\text { parents }\end{array}$ & $\begin{array}{l}\text { Average scores on exit on national } \\
\text { exams }\end{array}$ & $\begin{array}{l}\text { Percentage of students that completed SE in } \\
3 \text { years }\end{array}$ \\
\hline Average school & 10.67 & 10.60 & 10.93 & 0.35 \\
\hline Indifferent & 11.67 & 10.60 & 11.93 & 0.35 \\
\hline \multirow[t]{3}{*}{ schools } & 11.67 & 10.60 & 11.20 & 0.37 \\
\hline & 10.67 & 9.60 & 10.36 & 0.35 \\
\hline & 10.67 & 10.60 & 11.93 & 0.30 \\
\hline
\end{tabular}

Table 7

Trade-offs between inputs and outputs.

\begin{tabular}{|c|c|c|c|c|}
\hline & Average scores on entry & $\begin{array}{l}\text { Average no. of years in } \\
\text { school for the parents }\end{array}$ & $\begin{array}{l}\text { Average scores on exit } \\
\text { on national exams }\end{array}$ & $\begin{array}{l}\text { Percentage of students that completed } \\
\text { SE in } 3 \text { years }\end{array}$ \\
\hline \multirow[t]{4}{*}{ Trade-offs } & 1 & 0 & 1 & 0 \\
\hline & 1 & 0 & 0.27 & 0.02 \\
\hline & 0 & -1 & -0.57 & 0 \\
\hline & 0 & 0 & 1 & -0.05 \\
\hline
\end{tabular}

Note that the trade-offs defined as improvement in inputs and consequent improvement in outputs are similar to upper bounds on the ratio of output to input weights, whereas trade-offs defined as deterioration in inputs and consequent deterioration in outputs correspond to lower bounds on the ratio of output to input weights.

Table 8 shows the results of the standard DEA model without weight restrictions, the standard DEA model with ARII, and the nonlinear model proposed in this paper. In all cases we report both constant and variable returns to scale (VRS) results, where all models are assessed in output oriented form.

Note that we did not address variable returns to scale models in Section 3. This is because most of the problems mentioned for CRS models do not persist when the underlying technology is VRS. In particular, since the VRS model imposes the sum of lambdas to be 1 (in its envelopment form), there will be always at least one efficient DMU (100\% efficiency score). The VRS model with ARII is, therefore more flexible preventing cases of unfeasible solutions and of no $100 \%$ efficiency scores. However, the problem of under-estimation of relative efficiency persists. To correct for this problem the new model presented in this paper can be applied to the VRS case as long as a new constraint (imposing the sum of lambdas to be one) is added to the envelopment formulation, or a new variable, free in sign, is added to the multiplier model. The multiplier model formulation for the case of VRS output oriented model is shown in (18).

$$
\min _{u, v, w, c}\left\{\begin{array}{l}
\sum_{i} v_{i} x_{i o}-w \mid c \sum_{r} u_{r} y_{r o}=1, c \sum_{r} u_{r} y_{r j}-\sum_{i} v_{i} x_{i j}+w \leqslant 0, j=1, \ldots, n \\
\sum_{r} u_{r} a_{r t}-\sum_{i} v_{i} b_{i t} \leqslant 0, t=1, \ldots, k, u_{r} \geqslant 0, v_{i} \geqslant 0, c \geqslant \varepsilon, w \text { is free }
\end{array}\right\}
$$

Table 8 shows that CRS and VRS models without weight restrictions have higher efficiency scores and small discrimination between units (particularly the VRS model, where 8 out of 18 units appeared efficient) than the corresponding models with ARII. Results in Table 8 also show that ARII within traditional DEA models under-estimate the relative efficiency of all the schools in both CRS and VRS technologies. On average the gains obtained for the CRS case are not very high (efficiency improved from an average of $79.98 \%$ to $80.82 \%$ ), but for the 
Table 8

Comparison of efficiency estimates in the presence of ARII.

\begin{tabular}{|c|c|c|c|c|c|c|}
\hline \multirow[t]{2}{*}{ DMU } & \multirow[t]{2}{*}{ Standard CRS without ARII } & \multicolumn{2}{|l|}{ CRS ARII } & \multirow[t]{2}{*}{ Standard VRS without ARII } & \multicolumn{2}{|l|}{ VRS ARII } \\
\hline & & Standard CRS with ARII & Relative efficiency & & Standard VRS with ARII & Relative efficiency \\
\hline 1 & 0.9514 & 0.7828 & 0.7875 & 0.9599 & 0.8072 & 0.8138 \\
\hline 2 & 0.8384 & 0.6944 & 0.7047 & 0.873 & 0.7055 & 0.7172 \\
\hline 3 & 0.9787 & 0.7901 & 0.7999 & 0.9844 & 0.83 & 0.8432 \\
\hline 4 & 0.9784 & 0.872 & 0.8813 & 1 & 0.9248 & 0.9382 \\
\hline 5 & 0.8367 & 0.835 & 0.8367 & 1 & 1 & 1 \\
\hline 6 & 0.9974 & 0.851 & 0.8595 & 1 & 0.8745 & 0.8852 \\
\hline 7 & 0.8382 & 0.6862 & 0.6948 & 0.8601 & 0.7399 & 0.7529 \\
\hline 8 & 0.9978 & 0.9188 & 0.9295 & 1 & 0.9347 & 0.9472 \\
\hline 9 & 0.876 & 0.7491 & 0.7494 & 0.9836 & 0.8686 & 0.8756 \\
\hline 10 & 0.924 & 0.7459 & 0.7486 & 0.9669 & 0.8048 & 0.8111 \\
\hline 11 & 1 & 1 & 1 & 1 & 1 & 1 \\
\hline 12 & 0.9394 & 0.7417 & 0.7544 & 0.9576 & 0.7719 & 0.7876 \\
\hline 13 & 0.8756 & 0.7525 & 0.7679 & 0.9669 & 0.8388 & 0.8618 \\
\hline 14 & 0.9486 & 0.7102 & 0.72 & 1 & 0.7115 & 0.7219 \\
\hline 15 & 0.8278 & 0.7164 & 0.7235 & 0.854 & 0.7685 & 0.7795 \\
\hline 16 & 1 & 0.839 & 0.857 & 1 & 0.894 & 0.9175 \\
\hline 17 & 1 & 0.9002 & 0.9165 & 1 & 0.9443 & 0.9649 \\
\hline 18 & 0.9382 & 0.8102 & 0.8172 & 0.9599 & 0.8574 & 0.8679 \\
\hline Average & 0.9304 & 0.7998 & 0.8082 & 0.9648 & 0.8487 & 0.8603 \\
\hline
\end{tabular}

Table 9

Targets of school 4 by models (14) and output oriented form of (9).

\begin{tabular}{|c|c|c|c|}
\hline & Original value of school 4 & Targets with nonlinear model (14) & Targets with Podinovski's trade-off model (9) \\
\hline No. of pupils on entry & 149 & 133.1 & 138.77 \\
\hline Scores on entry & 1575.8 & 1575.8 & 1575.8 \\
\hline No. of years in school for the parents & 1554.07 & 1554.07 & 1554.07 \\
\hline Scores on exit on national exams & 1714.02 & 1944.79 & 1965.5 \\
\hline Students that completed in 3 years & 59 & 66.94 & 67.65 \\
\hline
\end{tabular}

VRS case the efficiency improvement is more marked (average efficiency improved from $84.87 \%$ to $86.03 \%$ ). On an individual basis school 16 was the one gaining more with a correct estimation of its relative CRS and VRS efficiency. Its CRS efficiency score passed from $83.9 \%$ to $85.7 \%$, and its VRS efficiency score passed from $89.4 \%$ to $91.75 \%$. Clearly in any other contexts apart from the school context, we should expect similar, if not higher, gains in efficiency when the correct model for assessing relative efficiency in the presence of ARII is used.

Using the output oriented modified trade-off model (14) we can assess the radial targets of DMUs as $\left(X_{0}, \beta^{*} Y_{o}\right)$ or we can use (16) to arrive at the targets of each school. As an example, the targets of school 4 obtained from model (14) and Podinovski's (2004) trade-off model (9) in output orientation form is as follows (see Table 9).

Note that in this case there is a slack on input 1 and the output targets are radial (there are no slacks in this case). The efficiency value from model (14) is equal to $88.13 \%$, and corresponds to the reciprocal of the proportional expansion suggested for all output levels. Note that using the trade-offs model (9) of Podinovski (2004), the efficiency estimate would be lower, and equal to 87.2\%, corresponding also to the reciprocal of the proportional expansion in outputs.

\section{Conclusion}

In this paper we addressed the problems that traditional CRS DEA models with weight restrictions of the type ARII may have. In particular these models may result in infeasible solutions for some DMUs, in under-estimation of relative efficiency scores, and in all DMUS being inefficient. We show through an illustrative example that these problems happen because the standard DEA model with ARII (or indeed its dual corresponding to the model with linked trade-offs) cannot assess relative efficiency since the constraints imposing maximum efficiency to be one in fact limit the search for the weights that show the unit in the best possible light. This problem has been addressed before by Podinovski and Athanassopoulos (1998) in relation to absolute weight restrictions. We proposed an alternative nonlinear model that can successfully measure relative efficiency in the presence of ARII or linked trade-offs and we discussed some attributes of the proposed model. This model is inspired on the idea of measuring relative efficiency through the Maximin model. The model proposed has been applied to a sample of secondary schools, to illustrate its use in a real-world context.

\section{Appendix A}

Proof of equivalence between the maximum model (1) with ARII and models (11) and (12):

- Proof of equivalence between model (1) with ARII and model (11). Consider the Maximin model (1) where ARII (7) are included in the model. Let us define the positive variable $c=m a_{j} x \sum_{r} u_{r} y_{r j} / \sum_{i} v_{i} x_{i j}$. The definition of $c$ implies that $\left(\sum_{r} u_{r} y_{r j} / \sum_{i} v_{i} x_{i j}\right) \leqslant c$. So we obtain model (11) from the Maximin model (1) with ARII (7) as follows: 


$$
\begin{array}{lll}
\max _{(u, v) \in R^{+}} \frac{\sum_{r} u_{r} y_{r o}}{\sum_{i} v_{i} x_{i o}} / c & \max _{(u, v) \in R^{+}} \frac{\sum_{r} u_{r} y_{r o}}{c \sum_{i} v_{i} x_{i o}} \\
\sum_{r} u_{r} y_{r j} & \Longleftrightarrow \quad \forall j & \sum_{r} u_{r} y_{r j}-c \sum_{i} v_{i} x_{i j} \leqslant 0 \forall j \\
\sum_{i} v_{i} x_{i j} & \leqslant j \\
\sum_{r} u_{r} a_{r t}-\sum_{i} v_{i} b_{i t} \leqslant 0 & \sum_{r} u_{r} a_{r t}-\sum_{i} v_{i} b_{i t} \leqslant 0
\end{array}
$$

- Proof of equivalence between model (11) and model (12)

Starting from model (11) and defining $t=1 /\left(c \sum_{i} v_{i} x_{i o}\right), u_{r}^{\prime}=t u_{r}$ and $v_{i}^{\prime}=t v_{i}$, by multiplying both sides of the constraints by $\mathrm{t}$ we can obtain model (12) from model (11) as follows:

$$
\begin{aligned}
& \max _{(u, v) \in R^{+}} \sum_{r} t u_{r} y_{r o} \quad \max _{\left(u^{\prime}, v^{\prime}\right) \in R^{+}} \sum_{r} u_{r}^{\prime} y_{r o}
\end{aligned}
$$

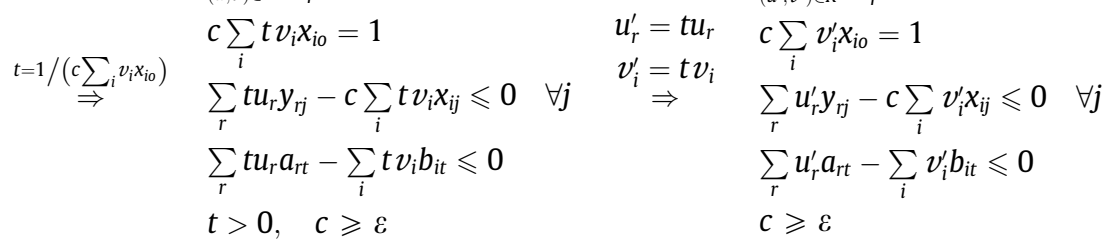

\section{References}

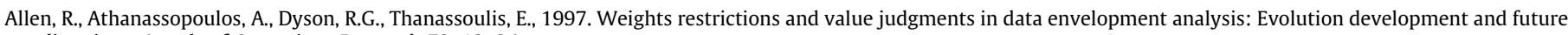
directions. Annals of Operations Research 73, 13-34.

Belton, V., Stewart, T.J., 2002. Multiple Criteria Decision Analysis: An Integrated Approach. Kluwer Academic Publishers, USA

Charnes, A., Cooper, W.W., Rhodes, E., 1978. Measuring the efficiency of decision making units. European Journal of Operational Research 2, $429-444$.

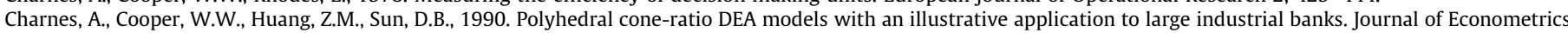
46, 73-91.

Cooper, W.W., Thompson, R.G., Thrall, R.M., 1996. Extensions and new developments in DEA. Annals of Operations Research 66, 3-45.

Dyson, R.G., Thanassoulis, E., 1988. Reducing weight flexibility in data envelopment analysis. Journal of the Operational Research Society (39/6), 563-576.

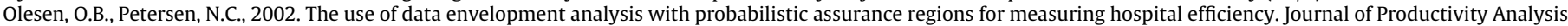
$17,83-109$.

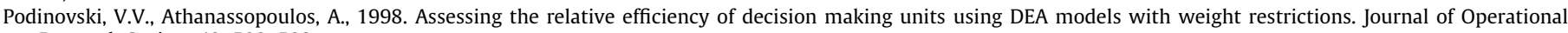
Research Society 49, 500-508.

Podinovski, V.V., 1999. Side effects of absolute weight bounds in DEA models. European Journal of Operational research 115, $583-595$.

Podinovski, V.V., 2001. DEA models for explicit maximization of relative efficiency. European Journal of Operational Research 52, $572-586$.

Podinovski, V.V., 2004. Production trade-offs and weight restrictions in data envelopment analysis. Journal of Operational Research Society 55, $1311-1322$.

Podinovski, V.V., 2005. The explicit role of weight bounds in models of data envelopment analysis. Journal of Operational Research Society, 1-11.

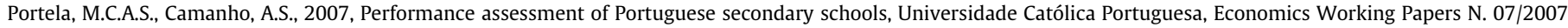
(available on-line at http://ideas.repec.org/p/cap).

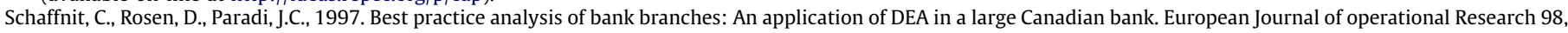
89-98.

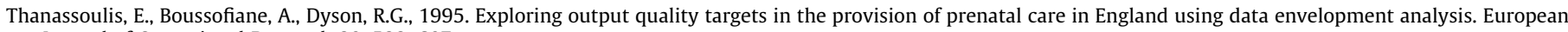
Journal of Operational Research 80, 588-607.

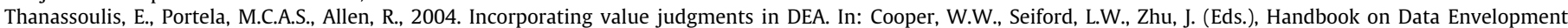
Analysis. Kluwer Academic Publishers, pp. 99-138.

Thompson, R.G., Singleton, F.D., Thrall, R.M., Smith, B.A., 1986. Comparative site evaluations for locating a high-energy physics lab in Texas. Interfaces 16 , 35-49.

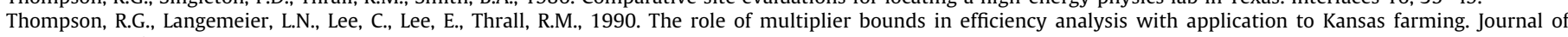
Econometrics 46, 93-108.

Thompson, R.G., Lee, E., Thrall, R.M., 1992. DEA/AR-efficiency of U.S. independent oil/gas producers over time. Computers and Operations Research 19/5, 377-391.

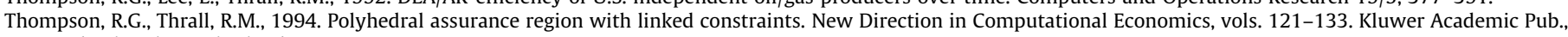
Dordrecht, The Netherlands.

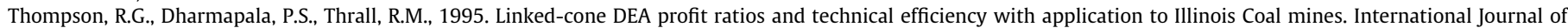
Production Economics 39, 99-115.

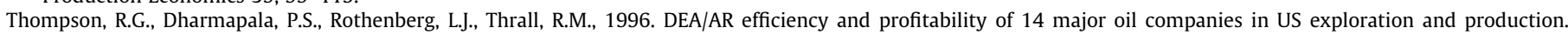
Computers \& Operations Research 23/4, 357-373.

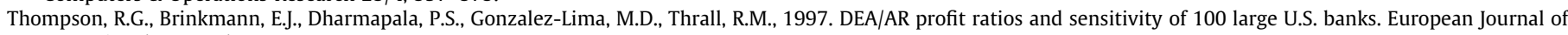
Operational Research 98, 213-229.

Tracy, D.L., Chen, B., 2005. A generalized model for weight restrictions in data envelopment analysis. Journal of Operational Research Society 56, 390-396. 